\title{
在分析化学课程中引入病毒及其检测方法的思考
}

雷杰 ${ }^{*}$, 包慧敏, 方彩云, 禁惠芝

复旦大学化学系, 上海 200433

摘要: 病毒学是以病毒为研究对象的一门基础生物学科。在分析化学课程中介绍病毒, 特别是 SARS-CoV-2 的结 构、功能和检测等, 可以使学生了解化学在病毒发现和检测过程中的作用。同时, 相关知识的引入不仅可以使学生 加深对分析化学中基本概念的理解, 而且可以使学生认识到分析化学的重要性和价值取向, 以及学科交叉的重要性 等, 还可以与课程思政教育结合, 进一步创造有意义的学习经历, 可谓一举多得。

关键词: 分析化学; COVID-19; 检测技术; PCR; ELISA; 学科交叉

中图分类号: G64; O6

\section{Introduction of Viruses and Its Detection Methods in the Course of Analytical Chemistry}

Jie Lei ${ }^{*}$, Huimin Bao, Caiyun Fang, Huizhi Fan

Department of Chemistry, Fudan University, Shanghai 200433, P. R. China.

Abstract: Virology is a basic biological science which takes virus as the research object. By introducing the structure and detection technology of virus, especially the structure and function of SARS-CoV-2 in the course of Analytical Chemistry, students can understand the role of chemistry in the development of virology. At the same time, the introduction of relevant knowledge can create meaningful learning experience; it can also be combined with course ideology and politics, so that students can realize the importance of basic concepts in Analytical Chemistry, value orientation of Analytical Chemistry, and the importance of interdisciplinarity.

Key Words: Analytical chemistry; COVID-19; Detection; PCR; ELISA; Interdisciplinarity

病毒性疾病占急性传染病的三分之二以上, 严重威胁着人类的生命和健康。2019年底爆发了新 型冠状病毒疾病(Coronavirus Disease 2019, COVID-19), 我国也将这种疾病称为新型冠状病毒肺炎, 简称新冠肺炎(Novel Coronavirus Pneumonia, NCP)。国际病毒分类委员会将 2019 年的新型冠状病毒 命名为 SARS-CoV-2 (Severe Acute Respiratory Syndrome Coronavirus 2); 世界卫生组织将其命名为 2019-nCoV (2019 novel coronavirus)。在短短几个月内, 疫情已经全球泛滥, 引起整个社会的广泛关 注。认识和控制新发病毒性疾病, 必需要依靠对病毒的全面认识和有效检测, 我们以此为切入点及 时在分析化学课程中引入病毒及检测方法的相关介绍, 引发学生思考分析检测技术在疾病早期诊断 和防控方面的价值。

首先, 结合新闻报道可以使学生了解到, 中国对疫情的及时控制来自医务人员的全力以赴、政 府和民间多部门的协同作战、群众的全力配合和自我管理能力等, 通过这一点可以进行课程思政教

收稿: 2020-04-30; 录用: 2020-08-02; 网络发表: 2020-08-05

“通讯作者, Email: jielei@fudan.edu.cn

基金资助: 复旦大学 2019 年度第二批教学研究与改革实践项目(2019A011); 教育部 2019 年第一批产学合作协同育人项目(201901102005) 
育, 让学生感受到中华民族的凝聚力。其次, 可以使学生深切认识到分析化学的价值取向之一: 把 学科发展同国家和民族发展紧紧联系在一起。第三, 病毒检测可以使学生更加深刻地认识分析化学 的概念: 利用物质一切可以利用的性质, 开发新的仪器设备, 建立表征测量的新技术和新方法。第 四, 可以使学生明白学科交叉的重要性, 分析化学的传统方法与新需求、新技术结合后, 同样会焕 发新的生命力, 比如 SARS-CoV-2 的检测就用到了传统的光学检测技术。第五, 结合 COVID-19 检 测中的 “假阴性” 问题, 可以使学生明白分析质量控制和分析质量评定的重要性。最后, 对化学类 专业的学生进行生物方面知识的科普, 可以使得学生对网络上的信息更加有鉴别力。

本文从化学工作者的角度, 对病毒及其检测技术进行科普, 文中尽量将实验原理和检测技术(试 剂盒)等阐述到分子层面, 展现化学专业的广泛应用价值, 让学生在学习的过程中有参与感。这也是 一个与学生共同探索的过程, 通过该过程可以创造有意义的学习经历 ${ }^{[1]}$ 。

\section{1 病毒介绍}

\section{1 病毒发现简史及分类}

19 世纪晚期, 病毒(Virus)这个词才和现在的理解基本一致, 当时荷兰的化学家阿道夫·迈耶 (Adolph Mayer)首次研究了烟草花叶病, 几年后, 丁努斯·拜耶林克(Martinus Beijerinck)在其研究基础 上发现这是一种当时未知的、比细菌还小的物质导致了烟草的这种病, 拜耶林克将这种 “有传染性 的活液” 称为病毒。当时的人们对病毒一无所知, 1923 年, 英国病毒学家弗雷德里克·特沃特 (Frederick Twort)甚至宣称: “人类无法确定病毒的本质” [2]。

20 世纪初, 科学家借助 X 射线解析蛋白质结构, 发现了酶的本质就是蛋白质。受此启发, 化学 家温德尔·斯坦利(Wendell Stanley)猜想: 病毒的本质是否也有可能是一种蛋白质呢? 于是他尝试结 晶烟草花叶病的病毒, 这是人们第一次可以不借助任何工具而用肉眼看到病毒 ${ }^{[3]}$ 。

1936 年, 英国科学家皮里(N. W. Pirie)和鲍登(F. C. Bawden)合作对自己精制出的烟草花叶病毒 结晶进行检测后发现, 该病毒含有相当量的硫和磷, 这两种元素在蛋白质中含量很少, 在核酸中含 量较大。经过进一步定量研究后发现, 蛋白质只占了该病毒组成的 $95 \%$, 还有 5\%是核酸。1939 年, 显微镜的发明让科学家们看到了烟草花叶病毒的形貌, 为约 $300 \mathrm{~nm}$ 的微小杆状结构 ${ }^{[3]}$ 。

随后, 科学家们逐渐了解了病毒的分子构成, 病毒主要由遗传物质和包裹在外的蛋白质衣壳 (capsid)组成, 对于复杂的病毒, 衣壳外面还有一层或几层富含脂质的外膜, 称为囊膜(envelope)。囊 膜的成分主要来自于宿主细胞, 一般是病毒在被感染细胞内或从被感染细胞表面 “出芽” 时从宿主 细胞获得的。某些病毒, 在囊膜和衣壳之间还有一层病毒特异的内膜蛋白; 有些病毒的衣壳里面还 有一层内衣壳 ${ }^{[4]}$ 。

病毒根据其遗传物质不同可以分为 DNA 病毒和 RNA 病毒。根据核酸类型, 病毒分为八大类 群, 即双链 DNA 病毒(double strand, dsDNA)、单链 DNA 病毒(single strand, ssDNA)、DNA 和 RNA 逆转录病毒、双链 RNA 病毒(dsRNA)、负单链 RNA 病毒 $(-s s R N A)$ 、正单链 RNA 病毒 $(+s s R N A)$ 、 裸露 RNA 病毒和类病毒。病毒按宿主种类不同又可分为动物病毒、植物病毒和细菌病毒(喍菌体) ${ }^{[5]}$ 。

\section{2 冠状病毒}

冠状病毒(coronavirus, CoV)的外形状如王冠, 并由此得名。冠状病毒属于正单链 RNA 病毒(与 mRNA 相似, 可以直接被核糖体翻译出蛋白质), 目前发现的冠状病毒有十几种, 为多形态, 略呈球 形, 直径 80-160 nm, 有囊膜, 囊膜表面覆有长 12-24 nm 的突起(即纤突), 突起末端呈球形, 突起 之间有较宽的间隙 $[6]$ 。

严重急性呼吸综合症(Severe Acute Respiratory Syndrome, SARS)的病原体, 称之为 SARS 冠状 病毒。2002 年 11 月发现的 SARS 冠状病毒被命名为 SARS-CoV。2019 年底发现的新型冠状病毒被 命名为 SARS-CoV-2, 平均直径 $100 \mathrm{~nm}$ 左右, RNA 链大小约 $30000 \mathrm{bp}$ (base pair) ${ }^{[7]}$ 。研究表明, SARS-CoV-2 与 SARS-CoV 的基因组序列相似度为 $73.4 \%{ }^{[7]}$ 。对于 SARS-CoV 的研究已经持续多年, 
目前关于 SARS-CoV-2 的研究仍在进行中, 很多论文暂时发表在预印本网站, 因此我们在教学中结 合已有文献对 SARS-CoV 和 SARS-CoV-2 进行介绍。

SARS-CoV 病毒粒子内部由 RNA 和核衣壳蛋白(nucleocapsid protein, $\mathrm{N}$ 蛋白)组成, 呈螺旋式结 构, 直径 9-16 nm。RNA 为核糖核酸, 基本组成单元为核苷酸, 核苷酸又是由含氮碱基、核糖和磷 酸组成, RNA 碱基主要有 4 种, 即腺嘌呤 $(A) 、$ 鸟嘌呤 $(G)$ 、胞嘧啶 $(C)$ 、尿嘧啶 $(U)$ 。 SARS-CoV 的 RNA 链可以编码结构蛋白、非结构蛋白和一些辅助蛋白, 是 RNA 病毒中基因组最长的病毒。其链 上包含 6-12 个开放阅读框(Open Reading Frame, ORF), 5’ 末端有帽结构, 3’末端有多聚腺苷酸(PolyA) 尾。5, 端开始约 $2 / 3$ 的基因区域编码酶蛋白, 后 $1 / 3$ 的区域依次编码纤突蛋白(spike protein, $\mathrm{S}$ 蛋白)、 包膜蛋白(envelope protein, $E$ 蛋白)、膜蛋白(membrance protein, $M$ 蛋白)、 $N$ 蛋白和一些辅助蛋白。 非结构蛋白基因散布于结构蛋白基因之间。在 SARS-CoV 和 SARS-CoV-2 的 E、M 和 N 蛋白的结构 基因比对中, $\mathrm{E}$ 蛋白基因的相似度最高, 可达到 $90 \%$ 以上, $\mathrm{M}$ 和 $\mathrm{N}$ 蛋白基因的相似度分别为 $85.6 \%$ 和 $88.8 \%$ 。SARS-CoV-2 一直在进行基因重组中, 截止 2020 年 7 月中旬, 已经有上万条 SARS-CoV-2 的基因序列上传至 GenBank 等共享平台 ${ }^{[8]}$ 。

SARS-CoV 的囊膜由双层脂质组成, $M$ 蛋白和 $\mathrm{E}$ 蛋白是囊膜形成所必需的成分, $\mathrm{M}$ 蛋白由 221 个氨基酸残基组成, $\mathrm{E}$ 蛋白是最小的结构蛋白, 由 76 个氨基酸残基组成, 其中 12-34 个残基存在跨 膜区域。SARS-CoV 的 $\mathrm{N}$ 蛋白是一种碱性蛋白, 由 422 个氨基酸残基组成, 相对分子质量为 46030 , 编码 $\mathrm{N}$ 蛋白的基因为 $1269 \mathrm{bp}, \mathrm{N}$ 蛋白与病毒 RNA 紧密结合, 通过螺旋堆积来保护病毒的 RNA。 SARS-CoV 的 S 蛋白的分子量约 $180 \mathrm{kDa}$, 由 1255 个氨基酸残基组成。 $\mathrm{S}$ 蛋白是一种横跨膜的糖蛋 白, 翻译后的 $\mathrm{S}$ 蛋白被切割成 2 个亚单元, 氨基端多肽为 $\mathrm{S} 1$ 亚单元, 它形成了病毒表面刺突的球 形头部; 羧基端多肽为 S2 亚单元, 它形成了与病毒表面膜蛋白结合的杆状部分。 $\mathrm{S}$ 蛋白是病毒的主 要抗原, 具有受体结合活性和膜融合活性, 前者决定了病毒可以感染哪些细胞, 后者可以使病毒进 入细胞, $\mathrm{S}$ 蛋白是感染后中和抗体的主要靶标, 也是病毒检测、药物开发和疫苗设计的重点。SARS-

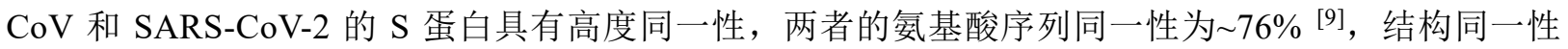

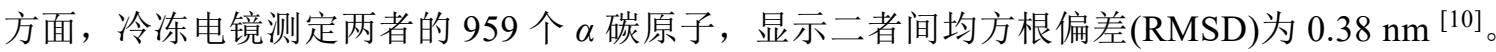

\section{2 病毒检测技术概述}

基于以上在分子层面上对病毒的认识, 就可以从分析化学的角度有针对性地设计相应的检测方 法。目前常用的病毒检测技术包括: 电镜观察技术、免疫学(血清学)检测技术、分子生物学检测技术 和生物芯片检测技术。

\section{1 电镜技术}

电镜观察首先需要在细胞中培养病毒, 再经超速离心等将病毒浓缩后, 经复杂的制样和切片过 程, 使得样品厚度在 10-100 $\mathrm{nm}$ 之间。观察病毒最常用的是负染技术和免疫电镜技术。负染是指利 用对电子散射力强的重金属盐在样品四周的堆积而加强样品外围的电子密度, 使样品显示负的反差, 祄托出样品的形态和大小。例如, 将病毒悬液经高度浓缩和纯化后, 借助磷铇酸负染后, 可用高分 辨率电子显微镜直接观察, 根据大小、形态等可初步判断病毒属哪一科 $[8]$ 。免疫电镜技术将免疫学 中的特异性抗原抗体反应与电镜技术结合, 另外还可以进行免疫胶体金标记。冷冻电镜用于溶液中 生物分子结构的高分辨率测定, 主要步骤为冷冻分子样品、电镜二维成像、软件三维重构, 目前已 有冷冻电镜用于 SARS-CoV-2 病毒检测的研究报道 ${ }^{[10,11]}$ 。但是这种方法中如果有过量的抗体等存在, 病毒可能会被掩盖, 导致出现假阴性结果。另外, 高分辨率电子显微镜设备比较昂贵, 检测结果还 依赖于显微镜操作者的技能等，不利于大规模流行病学调查。

\section{2 免疫学检测技术}

抗原(antigen)是指能够刺激机体产生免疫应答, 并能与免疫应答产物(抗体)和致敏淋巴细胞在体 内外结合, 发生免疫效应的物质。抗体 (antibody) 是指机体的免疫系统在抗原的刺激下, 由淋巴细胞 
等增殖分化成的浆细胞所产生的、可与相应抗原发生特异性结合的免疫球蛋白。一种抗原可能有具 有诱导产生多个抗体的空间结构, 即具有多个抗原决定簇。抗原和抗体的反应是其分子表面的非共 价相互作用特异性结合, 因此是可逆性的。通常情况下, 抗原只与它自己(或者具有相同抗原决定簇 的抗原)诱导产生的抗体发生反应, 因此免疫反应具有高度的专一性。用于病毒检测的免疫球蛋白有 $\operatorname{IgG}$ 和 IgM 两种, IgG 抗体是抗体中分子量较小的一种, IgM 抗体是抗体中分子量较大的一种 ${ }^{[12]}$ 。 冠状病毒的免疫学检测技术利用的是病毒 $\mathrm{N}$ 蛋白和 $\mathrm{S}$ 蛋白等的抗原性, 检测的目标主要是蛋白质。 最常用的免疫学检测技术包括酶联免疫吸附法、免疫胶体金标记法和生物芯片检测技术等。

\subsection{1 酶联免疫吸附法}

酶联免疫吸附法(Enzyme-Linked Immunosorbent Assay, ELISA)利用了酶的放大作用, 使得免疫 检测灵敏度大大提高。该方法的基本步骤是, 首先将待测样本(抗原)结合在固相载体(酶标板)上的小 孔内, 然后加入病毒特异性的抗体与酶(比如辣根过氧化物酶、碱性磷酸酯酶等)结合成的偶联物, 抗 原和抗体特异性结合后, 再加入酶的底物(比如邻苯二胺、四甲基联苯胺等), 在酶的作用下, 无色底 物变化为有色的产物, 可以通过肉眼观察或酶标仪(专用于测读 ELISA 结果的分光光度计)等测定颜 色深浅或吸光度, 测定结果与待测抗原的含量成正比, 据此可以对抗原进行定性和定量测定。以上 为直接 ELISA, 另外还有双抗体夹心 ELISA, 顾名思义, 此法多了一个步骤, 具体连接顺序为 “酶 标板-抗体-待测抗原-酶标记的抗体”，双抗体夹心 ELISA 可以提高检测的特异性。

选择鲁米诺(3-氨基苯二甲酰肼)为酶的底物时, 因为鲁米诺与双氧水会一起被辣根过氧化物酶 氧化生成不稳定的中间体, 此中间体再进一步转化成氨基苯甲酸时, 会发出苂光, 此时的 ELISA 即 为化学发光法检测, 在此体系中加入苯酚及荎酚类化合物, 还可以提高化学发光的灵敏度。另外还 可以采用苂光底物, 这时就需要采用荧光检测器。从化学工作者的角度进行研究, 可以探究底物的 具体显色反应路径, 以及最佳反应条件, 还可以从仪器分析的角度设计酶标仪, 优化其使用条件等。 有机化学家则可以设计合成针对不同酶的底物, 来提高检测的灵敏度等。这就是化学可以助力生物 学之处, 也是学科交叉的突破口, 下文中 PCR 等技术中也存在类似的思路。

ELISA 可以对病毒抗原或抗体进行检测。例如, 可以在杆状细菌等中表达出重组的 $\mathrm{N}$ 蛋白、M 蛋白等, 将其纯化后, 可在 IgG ELISA 法检验中作为 SARS-CoV-2 抗体的抗原, 这种方法可以作为 SARS-CoV-2 诊断和血清流行病分析的有效工具。

\subsection{2 免疫胶体金标记法}

金纳米颗粒形成的胶体溶液即为胶体金，在碱性条件下，金纳米颗粒表面带负电荷，可以与抗 原或者抗体所带正电荷之间因静电吸引而牢固结合。金纳米颗粒聚集达到一定密度时, 就会出现肉 眼可见的粉红色斑点。免疫胶体金标记法主要是将特异的抗体交联到试纸条上或有颜色的物质上, 当此抗体与特异性抗原结合后, 再和胶体金标记的特异性抗体进行反应时, 就形成了带有胶体金颜 色的三明治结构。如果没有抗原则没有颜色。免疫胶体金标记法由于具有操作简单、无需任何专用 仪器、无需特殊培训操作人员、检测结果直观和方便现场使用等特点, 成为近些年体外诊断试剂发 展的趋势。目前市场上可以迅速得到检测结果, 如验孕般简单的检测试剂多使用免疫胶体金法。该 检测法是仪器微型化、试纸化的一个成功的典范, 对化学专业的学生是一个很好的教育案例。

免疫学检验的局限性在于, 抗原抗体反应的专一性导致了每种被检测对象都需要开发专门的检 测试剂盒; 处理检测对象时, 其中蛋白质的抗原性很容易被破坏, 造成检测失败; 有些检测对象, 如转基因产品, 其外来插入的基因基本不表达蛋白, 或者是表达量很低, 从而导致无法进行检测。

\section{3 分子生物学检测技术}

分子生物学检测技术是在人们对基因结构以及基因的表达和调控等生命本质问题的认识日益深 刻的基础上产生的。分子生物学检测技术的检测目标是核酸, 主要包括: PCR 技术、核酸杂交技术 等, 下面对最常用的 PCR 技术进行介绍。 


\subsubsection{PCR 技术的基本原理}

1983 年, 美国科学家 Kary Mullis 等首先提出 PCR 的想法, 并于 1985 年申请了 PCR 的专利。 Kary Mullis 也因此而荣获 1993 年的诺贝尔化学奖。PCR 技术的发明得益于耐热性 DNA 聚合酶的发 现, DNA 聚合酶最早于 1955 年发现, 但是耐热性 DNA 聚合酶是 1976 年才从温泉中的细菌(Thermus aquaticus) 分离出来的, 简称为 $T a q$ 酶( $T a q$ polymerase)。

PCR 是聚合酶链式反应(Polymerase Chain Reaction)的简称, 是一种酶促化学反应, 用于短时间 内快速、大量扩增特定基因片段(携带有遗传信息的 DNA 或 RNA 序列)的分子生物学技术。传统 PCR 技术在高温变性 $\left(95^{\circ} \mathrm{C}\right)$ 时, 双链模板 DNA 碱基间的氢键发生断裂, 形成单链 DNA 作为下一步反应 的模板。低温退火 $\left(60^{\circ} \mathrm{C}\right)$ 时, PCR 扩增引物与单链 DNA 模板按照碱基互补配对原则进行序列配对, 形成模板-引物复合物。适温延展 $\left(72{ }^{\circ} \mathrm{C}\right)$ 是在 $\mathrm{Taq}$ 酶的催化作用下, 以三磷酸脱氧核苷酸 (deoxynucleotide triphosphates, dNTPs)为反应原料, 以目标序列为模板, 按碱基互补配对原则与半保 留复制原理, 以引物为固定起点, 按 5, 到 3'方向延伸, 生成一条全新的 DNA 模板互补链, 从而使 单链 DNA 又重新恢复到双链。

以上三步作为一个循环重复进行, 由于扩增是呈指数级进行的, 以上步骤每个需要 2-4 min, 2-3 h 即可完成整个扩增任务。PCR 扩增产物的分析方法包括: 凝胶电泳分析、酶切分析、分子杂交 和核酸序列分析等 ${ }^{[13]}$ 。

\subsubsection{PCR 技术的反应体系}

一般的 PCR 实验需要在可靠的、无污染的专门实验室进行, PCR 的反应体系包括以下几个主要 成分: 模板 DNA、扩增引物、缓冲液 $\left(\right.$ 含 $\mathrm{Mg}^{2+}$ )、底物、耐热 DNA 聚合酶等。将以上主要成分放入 PCR 试管, 在 PCR 扩增仪上进行。

PCR 反应的模板(template)可以是 DNA, 也可以是 RNA, 当使用 RNA 做模板时, 需先将 RNA 经过逆转录生成 cDNA (complementary DNA), 然后再进行 PCR 反应。模板 DNA 中需尽量避免有抑 制 PCR 的杂质存在, 比如蛋白酶、可与 DNA 结合的杂蛋白等。同时, 模板 DNA 的量不能太高, 否则会影响到 PCR 扩增的效果。

扩增引物(primers)为长度 15-30 bp 的单链葟聚核苷酸片段, 需与待扩增的目标 DNA 片段两侧 互补。引物的设计要考虑多方面的因素, 需根据实际情况具体分析, 现在已经有计算机软件可以帮 助设计引物。底物 dNTPs 为相同浓度的四种脱氧核苷(dATP、dDTP、dTTP、dCTP)的混合液。

最常用的耐热 DNA 聚合酶为 $T a q$ 酶, 能在 $70-75^{\circ} \mathrm{C}$ 生长。 $T a q$ 聚合酶具有 5 , 到 3 ' 聚合酶活力 和 5'到 3'外切酶活力, 而无 3'到 5'外切酶活力, 因而在 PCR 反应中如发生某些碱基的错配, 该酶 没有校正功能, $T a q$ 聚合酶的配错几率为 $2.1 \times 10^{-4}$ 。现在已经出现具有校对功能的高保真 DNA 聚 合酶, 如 Taq plus I、II 等 DNA 聚合酶。所有耐热 DNA 聚合酶活性的维持都需要二价阳离子, 通常 是 $\mathrm{Mg}^{2+}[13]$ 。

\subsubsection{PCR 技术的发展}

传统 PCR 技术容易引起污染, 而且操作步骤繁多, 人工操作劳动强度大, 不适合样品量大的商 业检测。目前, 已发展出许多新型的 PCR 技术, 例如: 逆转录 PCR、多重 PCR、原位 PCR 和定量 PCR 等。RNA 病毒的检测需要逆转录 PCR (reverse transcription PCR, RT-PCR), 也就是将 RNA 的 逆转录和与其互补的 cDNA 的 PCR 相结合。首先需要提取出病毒中的 RNA, 以其作为模板, 利用 逆转录酶反转录合成 cDNA, 再通过 PCR 技术, 将痕量的 cDNA 扩增成大量的双链 DNA 的过程。 多重 PCR (Multiplex PCR) 是在扩增体系中使用两对及两对以上的扩增引物, 使得多个目的核酸片段 能同时扩增, 可以做到同时检测多种病毒。

实时荧光定量 PCR (real-time quantitative PCR, qPCR) 通过对扩增反应中每一个循环产物的荧光 信号的实时监测, 从而实现对起始模板的定量。由于该技术是在同一 PCR 试管内完成扩增、荧光探 针杂化和信号检测, 大大降低了污染的可能性, 提高了检测的准确度, 而且荧光探针检测比常规的 
凝胶染色检测的灵敏度也大幅提高，真正做到了快速、准确、灵敏。

$q P C R$ 所使用的荧光物质可分为两种: 荧光探针和荧光染料。TaqMan 苂光探针是一个寡核苷酸, 可以与目的基因特异性结合, 其两端分别标记一个报告荧光基团(reporter, $\mathrm{R}$ )和一个淬灭苂光基团 (quencher, Q)。探针完整时, $\mathrm{R}$ 发射的荧光信号被 Q 吸收; 在 PCR 扩增时, 引物和探针同时结合到 模板上, 当扩增延伸到探针结合位置时, $\mathrm{Taq}$ 酶的 5'-3'外切酶活性将探针酶切降解, 使 $\mathrm{R}$ 和 $\mathrm{Q}$ 分 离, 从而使得 $\mathrm{R}$ 发射的苂光能被苂光检测系统检测到, 即每扩增一条 DNA 链就有一个苂光分子形 成, 实现了荧光信号的积累与 PCR 产物形成的完全同步。SYBR Green 荧光染料能结合到 DNA 双 螺旋上。在加入过量 SYBR 荧光染料的 PCR 反应体系中, 掺入 DNA 双链的 SYBR 荧光染料会发射 荧光信号, 而未掺入 DNA 链的染料不会发射苂光信号, 从而保证苂光信号的增加与 PCR 产物的增 加完全同步。

环介导等温扩增技术(Loop-mediated Isothermal Amplification, LAMP)于 2000 年由日本学者 Notomi $\mathrm{T}$ 等提出, 是一种新的核酸等温扩增技术, 其特点是针对靶基因的 6 (或 8) 个特定部位设计 4 (或 6) 种特异性引物。以四链引物体系为例, 其设计是在靶基因中选取六个区域, 从 5, 端到 3’端依 次为 B3、B2、B1、F1c、F2c、F3c, 然后根据这六个区域设计四条引物。前内引物 FIP (forward inner primer)由 F1c 和 F2 区域构成, F1c 与目标序列的 F1c 区域具有相同的序列, F2 与目标序列的 F2c 序列互补; 后内引物 BIP (backward inner primer) 由 B1c 和 B2 区域构成, B1c 与目标序列的 B1 序列 互补, B2 与目标序列的 B2 区域序列相同; 前外引物 F3 由 F3 区域构成, 与目标序列中的 F3c 序列 互补; 后外引物 B 3 由 B 3 区域构成。内引物和外引物的序列不同, 分别对应目标 DNA 的正义链和 反义链。在 LAMP 反应的起始阶段, 内引物和外引物均参与其中, 产生双哑铃结构 DNA, 用于引发 循环扩增反应, 当反应进入循环扩增阶段, 只有内引物参与反应。反应在链置换 DNA 聚合酶的作用 下, 60-65 ${ }^{\circ} \mathrm{C}$ 恒温扩增 15-60 min 即可实现 $10^{9}$ 倍左右的核酸扩增, 链置换 DNA 聚合酶可以实现 DNA 链的置换反应, 所以不再需要传统 PCR 的高温变性过程。LAMP 的最大特点是简便快速, 对 仪器和人员要求低。扩增反应产生大量副产物(焦磷酸镁)形成乳白色沉淀, 进行肉眼观测或用浊度 仪进行检测, 简单快捷。此外, 也可以在反应管中加入苂光染料, 观察颜色进行定性分析或通过定 量 PCR 仪进行定量分析。LAMP 检测 RNA 病毒时, 只需要在反应体系中加入反转录酶即可 ${ }^{[14]}$ 。

\section{4 生物芯片检测技术}

生物芯片是一种微型多参数生物传感器, 通过半导体光刻加工等微缩技术, 在一微小的固体载 体表面固定大量的分子识别探针, 构建微分析单元和系统。该技术将生命科学中许多不连续的分析 过程, 如样品制备、生物化学反应和定性、定量检测等集成于芯片上, 使这些分析过程连续化和微 型化。

基因芯片是生物芯片的一种, 又称为 DNA 芯片, 是指将大量核酸探针以预先设计的方式固定 在芯片上, 组成密集的分子阵列, 然后与荧光标记的靶分子进行杂交, 最后通过扫描仪及计算机进 行综合分析, 进而得到样品中基因的数量和序列等信息。PCR 芯片属于基因芯片中的微流路芯片, 最早的 PCR 芯片仍采用普通 PCR 技术, 将微流通道系统分成三个温度区, 由芯片下方放置三块恒 温铜块作为热源 ${ }^{[15]}$ 。现在的 PCR 芯片技术很多已经采用了恒温 PCR 技术, 比如前文提到的 LAMP 技术。

\section{SARS-CoV-2 的检测}

\section{1 采样技术}

血清学检测的基本步骤为: 抽血 $\rightarrow$ 血清分离 $\rightarrow$ 抗原抗体卯育 $\rightarrow$ 检测。PCR 检测的基本步骤如下: 组织液氮破碎 $\rightarrow$ RNA 的提取纯化 $\rightarrow$ RNA 反转录 $\rightarrow$ PCR。所有的检测技术都起始于采样。

血清学检测时需要的是血液样本, 血液样本的采集比较容易规范, 而且血液样本含病毒量低或 者不含病毒, 可以大大降低医护人员被感染风险。目前, 检测 COVID-19 疑似病例的 PCR 技术使用 
的样本采集方法多为上呼吸道样本(咽拭子为主), 另外还有采集肺泡灌洗液、深部痰液和肛拭子等。 SARS-CoV-2 的传播能力较强, 因此个人防护应为生物安全三级(P3)实验室级别。COVID-19 本身就 是下呼吸道疾病, 严格来说应该采集肺泡灌洗液等做核酸检测, 但是这种取样方式不方便, 最方便 的方式还是咽拭子。有文献报道患者用药情况、采样管的差异和核酸提取方法不同等都会影响检测 结果 ${ }^{[16]}$ 。这就导致了新闻报道中多次提到的 “假阴性” 问题。从分析化学的角度来看, 除了采样问 题, 其他影响检测结果的因素还有很多, 如样本的保存、转运、预处理, 以及检验环境达标与否等。 总之, 检验前、检验中和检验后的质量控制都很关键 ${ }^{[17]}$ 。

\section{2 检测技术}

新型冠状病毒肺炎诊疗方案(试行第七版)中, 关于确诊标准规定, 疑似病例同时具备以下病源 或血清证据之一者可确诊：（1）实时苂光 RT-PCR 检测新型冠状病毒核酸阳性；(2) 病毒基因测序, 与已知的新型冠状病毒高度同源; (3) 血清新型冠状病毒特异性 IgM 抗体和 IgG 抗体阳性; 血清新 型冠状病毒特异性 IgG 抗体由阴性转为阳性或恢复期较急性期 4 倍及以上升高 ${ }^{[18]}$ 。

对于新出现的病毒, 只要基因测序得到其基因序列, 对应的 PCR 技术就很容易研发 ${ }^{[7]}$ 。PCR 鉴 定依赖于该病毒 RNA 序列上的高度保守的序列, 即使病毒再次发生变异, 这些高度保守的序列在 进化中基本保持不变。COVID-19 核酸检测试剂盒主要检测 SARS-CoV-2 RNA 序列上的开放阅读框 $1 \mathrm{ab}(\mathrm{ORF} 1 \mathrm{ab})$ 和 $\mathrm{N}$ 蛋白基因, 有的公司开发的产品还会同时检测另外的基因片段(如 $\mathrm{E}$ 蛋白基因 等) ${ }^{[19]}$ 。关于血清学检测, 国家药监局应急审批新型冠状病毒检测产品(2020 年 3 月 17 日发布)有胶 体金法和磁微粒化学发光法等, 主要针对 $\operatorname{IgM} / \mathrm{IgG}$ 进行检测, 如果对疑似新冠肺炎的病人进行血清 检测, 发现血液中存在大量的 IgM, 说明他刚刚感染了新冠病毒; 接着通过治疗, 这位病人再次进 行血清检测时, 发现 IgM 的数量减少, IgG 的数量增多, 说明他的体内对新冠病毒有了抵抗力和免 疫力。血清学检验是对病毒核酸检验的有效补充, 发病早期, 核酸检测的灵敏度高于血清抗体检测, 但发病后期抗体检测则较为灵敏 ${ }^{[20]}$ 。血清学检测还可以采用化学发光和免疫胶体金标记法, 针对 191 例 COVID-19 患者和 27 例非 COVID-19 的检测结果表明, 化学发光法的检出率要高于免疫胶体金 标记法 ${ }^{[21] 。}$

除了以上诊疗方案中的方法, 电镜技术也被用于 SARS-CoV-2 的检测, 例如国内外学者使用冷 冻电子显微镜观察到了 SARS-CoV-2 经灭活后的形貌, 并且捕捉到了该病毒侵染宿主细胞的一个重 要中间状态。此时病毒正处于识别和附着宿主细胞后, 准备与细胞发生融合 ${ }^{[10,11]}$ 。

\section{4 结语}

病毒及其检测方法相关知识的教学过程, 具有自发性、参与性、发展性和反思性的特点, 不仅 可以使学生由被动学习转变为主动参与, 有效地调动学生学习的积极性, 还对学生今后的学习、生 活和就业非常有帮助。总之, 该教学过程可以在分析化学课程中为学生创造有意义的学习经历, 具 体可以促进基础知识、应用、综合、人文维度、关心和学会学习六个维度的学习 ${ }^{[1]}$ 。

\section{参 考 文 献}

[1] L·迪·芬克. 创造有意义的学习经历一综合性的大小课程设计原则. 胡美馨, 刘颖, 译. 杭州: 浙江大学出版社, 2006 .

[2] 卡尔·齐默. 病毒星球. 第 2 版. 刘旸, 译. 广西: 广西师范大学出版社, 2019.

[3] 卢礼亚, 等著. 普通病毒学. 第 3 版. 王顺德, 等译. 上海: 上海科学出版社, 1987.

[4] 殷震. 动物病毒学基础. 长春: 吉林人民出版社, 1980 .

[5] 谢天恩, 胡志红. 普通病毒学. 北京: 科学出版社, 2015 .

[6] 孙淑芳, 王媛媛, 刘陆世, 姜雯, 彭程, 孙映雪. 中国动物检疫, 2013, 30 (6), 68 .

[7] Wu, F.; Zhao, S.; Yu, B.; Chen, Y. M.; Wang, W.; Song, Z, G.; Hu, Y.; Tao, Z. W.; Tian, J. H.; Pei Y. Y.; et al. Nature 2020, 579 (7798), 265. 
[8] 张文宏. 2019 冠状病毒病: 从基础到临床. 上海: 复旦大学出版社, 2020 .

[9] Hoffmann, M.; Kleine-Weber, H.; Schroeder, S.; Schroeder, S.; Kruger, N.; Herrler, T.; Erichsen, S.; Schiergens, T.; Herrler, G.; Wu, N. H.; et al. Cell 2020, 181, 271.

[10] Wrapp, D.; Wang, N. S.; Corbett, K. S.; Goldsmith, J. A.; Hsieh, C. L.; Abiona, O.; Graham, B. S.; McLellan, J. S. Science 2020, 367 , 1260.

[11] Liu, C.; Yang, Y.; Gao, Y. Z.; Shen, C. G.; Ju, B.; Liu, C. C.; Tang, X.; Wei, J. L.; Ma, X. M.; Liu W. L.; et al. BioRxiv. 2020, doi: $10.1101 / 2020.03 .02 .972927$

[12] 江世益, 张鲁雁. 免疫化学. 上海: 上海医科大学出版社, 1996.

[13] 刘戟, 曾凡才. 生物化学与分子生物学实验教程. 北京: 科学出版社, 2017.

[14］袁向芬，吕继洲，吴绍强，王巧黎，赵宏. 生物技术通讯, 2018, 34 (10), 64.

[15] 曹雪雁, 张晓东, 樊春海, 胡钧. 自然科学进展, 2007, 17 (5), 580.

[16] 里进, 叶光明, 陈良君, 王嘉俊, 李一荣. 中华检验医学杂志, 2020, 43 (3), 221.

[17] 莫茜, 秦炜, 傅启华, 关明. 中华检验医学杂志, 2020, 43 (3), 213.

[18] 中华人民共和国卫生健康委员会. 关于印发新型冠状病毒感染的肺炎治疗方案(试行第七版)的通知. [2020-03-03]. http://www.nhc.gov.cn/yzygj/s7653p/202003/46c9294a7dfe4cef80dc7f5912eb1989.shtml.

[19] 张晓元, 张艳艳, 张小刚, 刘霞, 陈勉, 刘飞, 张岱州, 凌沛学. 生物化学与生物物理进展, 2020, 47 (4), 275.

[20] 徐万洲, 李娟, 何晓云, 张才庆, 梅四青, 李从荣, 李艳, 程少华. 张平安. 中华检验医学杂志, 2020, 43 (3), 230.

[21] 唐鹏, 赵自武, 刘颖娟, 陈雅文, 彭定辉, 王冬, 李一荣, 谢文. 武汉大学学报(医学版), 2020, doi: 10.14188/j.1671-8852.2020.0131 